\title{
IL PARCO ARCHEOLOGICO NATURALISTICO DEL PORTO DI TRAIANO. \\ INTERAZIONI CON GLI ELEMENTI DEL PAESAGGIO
}

\author{
GABRIELLA STRANO \\ gabriella.strano@beniculturali.it \\ PARCo ARCHEOLOGICO DEL COLOSSEO \\ http://dx.doi.org/10.25267/Riparia_sup.2018.11.15
}

Quello che il nostro sguardo abbraccia varcando il cancello o già velocemente dall'alto del viadotto che si affaccia sul Sito Archeologico Naturalistico di Portus, è il paesaggio suggestivo di un luogo in cui i segni della storia ne fanno un tòpos senza tempo, in cui la materialità dei lacerti antichi ha assunto carattere di naturalità e la linea delle geometrie vegetali rivela un disegno preciso nella scelta delle essenze e dell'impianto. L'area archeologica, distante $30 \mathrm{~km}$ dal centro di Roma, è raggiungibile percorrendo l'asse autostradale Roma-Fiumicino o la via Portuense moderna che per molti tratti segue il percorso della strada antica, la via Campana, lungo la riva destra del Tevere. Il viadotto, che collega Roma e l'aeroporto Leonardo da Vinci con gli ambiti litoranei di Fiumicino e Ostia, crea a ovest il limite fisico tra il moderno e l'antico. Un limite poroso in cui le rispettive identità delle due parti rimangono riconoscibili. Il sipario vegetale formato dal filare di Eucaliptus piantati nel primo trentennio del 1900, nel periodo della bonifica della plaga pontina e ora a ridosso della carreggiata, tenta di creare uno schermo visivo e sonoro per isolare il Parco dall'urbanizzazione disordinata dell'intorno fortemente accelerata nel corso del XX secolo.

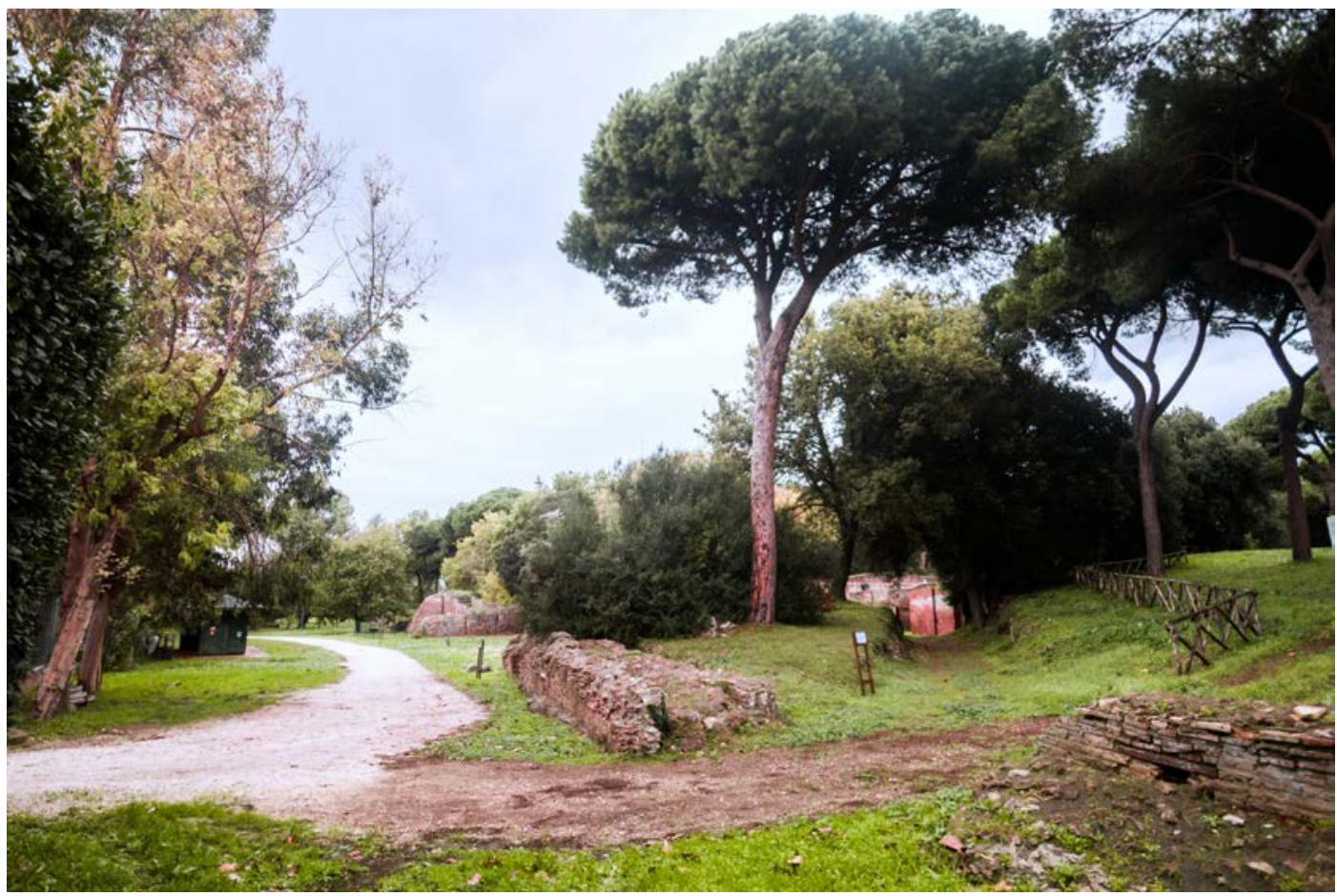

Fig. 1. Parco archeologico naturalistico del Porto di Traiano. 
Negli anni '50 del XX sec. fu costruito, a ridosso dell'antico Porto imperiale, il grande aeroporto di Roma e con il Piano Regolatore Generale del 1962 l'area litoranea a nord del Tevere è stata destinata alla realizzazione di infrastrutture, zone industriali, artigianali e commerciali. Dagli anni ' 90 sono stati realizzati, e ancora sono in corso di realizzazione, estesi insediamenti a carattere residenziale. Negli ultimi decenni il territorio ha subito dunque una vera e propria aggressione edilizia.

Il sito archeologico di Portus, con i suoi 33 ettari di area a verde rappresenta una sacca ambientale, un importante sistema da salvaguardare per lo sviluppo degli ecosistemi, l'abbondanza e la distribuzione degli organismi e la biodiversità nel contesto dell'ambiente protetto che racchiude. Le antiche vestigia sono immerse in una vegetazione semi naturale scaturita dalle piantumazioni antropiche dei primi anni del $\mathrm{xx}$ secolo e da un periodo di abbandono che ha permesso alla natura di riprendersi i suoi spazi ridando vita a un luogo di grande suggestione dove all'enorme valenza archeologica si accompagna quella naturalistica, sia per l'importante patrimonio arboreo che può a tutto titolo considerarsi storico, sia per la presenza di zone umide, fattore di attenzione per la difesa della biodiversità nel parco archeologico oggi e nel suo sviluppo futuro.

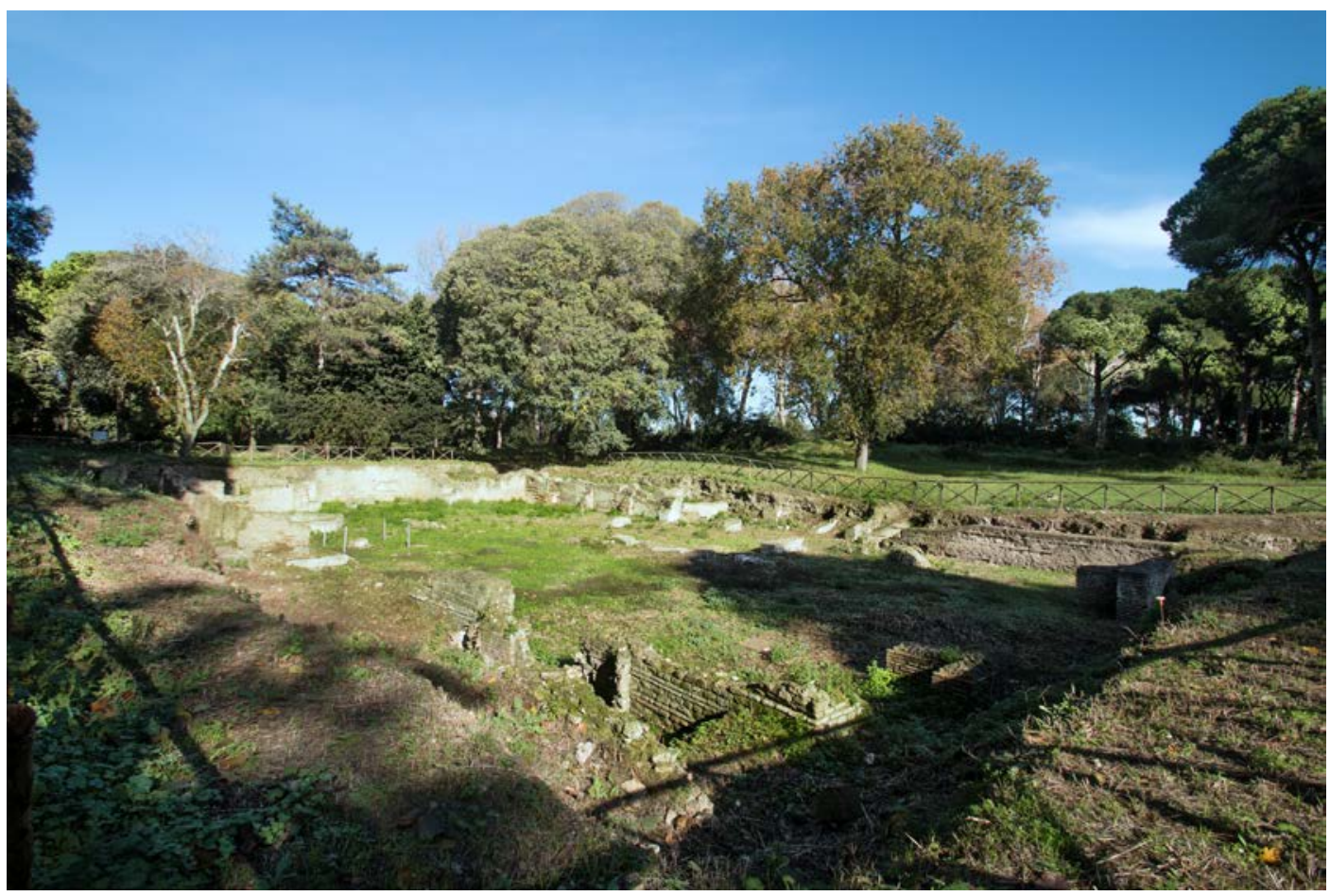

Fig. 2. Area della basilica (Foto F. TORTORA).

Il Sito è inserito all'interno della Zona 1 di riserva integrale della Riserva Naturale Statale del Litorale Romano e l'invaso del lago di Traiano, di natura artificiale, è classificato come Zona Umida ai sensi della Convenzione di Ramsar del 1974, ovvero area assimilabile a "stagni, paludi, torbiere, bacini naturali e artificiali permanenti con acqua stagnante o corrente dolce, salmastra". La zona di tutela d'interesse di Tipo 1 naturalistico paesaggistico e culturale coincide con il corridoio fluviale del Tevere, mentre le zone di Tipo 2 riguardano gli ambienti agricoli della bonifica. 
Il sistema della legislazione vincolistica esercita potere di tutela storico-archeologica, architettonica, paesaggistica e ambientale, finalizzata alla salvaguardia e valorizzazione del sito, inserito nel Piano Territoriale Paesaggistico della Regione Lazio. Una parte dell'antica darsena è individuata come Sito di Interesse Comunitario (SIC): Direttiva del Consiglio relativa alla Conservazione degli habitat naturali e seminaturali e della flora e della fauna selvatiche nota anche come Direttiva Habitat recepita in Italia dal 1997 e Zona a Protezione Speciale (ZPS) IT6030026 istituita ai sensi della direttiva comunitaria 79/409/CEE “Uccelli” e 2009/147/CE all'interno della Rete Natura 2000. Il Sito comprende oltre i propri confini "aree core" ovvero "aree di interesse naturalistico già sottoposte a vincoli e normative", caratterizzate nel suo intorno da un "area buffer", ovvero "serbatoi di naturalità a contatto con aree core con presenza di flora, fauna e vegetazione di notevole interesse conservativo e biogeografico".

Ostia Antica e Portus, che costituivano il sistema portuale antico, ora, insieme alla Necropoli dell'Isola Sacra, compongono un sistema archeologico di grande importanza storico-architettonico e paesaggistico. Anticamente la loro posizione era strategica per il controllo della foce del fiume Tevere e delle antiche saline, centri produttivi di primaria importanza. Il progressivo insabbiamento, già in atto fin dall'antichità, ha allontanato quello che fu il principale polo marittimo della Roma imperiale, di circa $3 \mathrm{~km}$ dal mare.

Il parco sorge infatti sul terreno di sedimentazione che in circa duemila anni si è depositato nell'antico bacino portuale. Nel 42 d.C. l'imperatore Claudio diede avvio alla costruzione del primo impianto posto a $3 \mathrm{~km}$ a nord dalla foce del Tevere, ampio circa 200 ettari. Fu scavato in parte nella terra ferma e in parte racchiuso verso mare da due grandi moli. Due canali artificiali assicuravano il collegamento con il mare e il Tevere. Il porto fu concluso e inaugurato nel 64 d.C. da Nerone, come risulta dalle notizie storiche e dalle monete commemorative.

L'impianto, fin dalla nascita, fu soggetto a grandi problemi di sicurezza e funzionalità. Come ci raccontano le fonti storiche incendi e il continuo progressivo insabbiamento resero da subito difficoltoso il transito delle navi e delle merci; gran quantità di detriti fluviali e marini si andavano a depositare lungo la costa aumentandone la linea e alzando $\mathrm{i}$ fondali. Le cronache del tempo ci parlano di squadre di dragatori costantemente al lavoro per alleggerire $\mathrm{i}$ fondali in modo che le navi potessero entrare senza pericolo.

La realizzazione della struttura portuale ebbe un grande impatto sulla morfologia del territorio: il litorale subì la trasformazione della linea di costa, si accentuarono gli spostamenti dei meandri nel tratto finale del Tevere e con il taglio nell'entroterra per la costruzione del canale di collegamento del porto con il Tevere si venne a formare l'Isola Sacra.

Dopo pochi decenni di attività, e in seguito probabilmente a un grande incendio, tra il 110 e il 117 d.C., l'imperatore Traiano iniziò le opere per l'ampliamento dell'impianto portuale e la costruzione del grande bacino esagonale. Monete commemorative sono datate 112 d.C. e uno scolio, menzionato dalle fonti, a commento di un verso di Giovenale recita: "Traiano restaurò , migliorandolo, il porto di Augusto e ne costruì più all'interno uno più sicuro che porta il suo nome" (satire, XII, 75).

In seguito alle scorrerie barbariche, che devastarono Roma e tutta l'area tiberina, la funzionalità del porto fu limitata concentrando il traffico mercantile in alcuni ambiti più protetti, arroccandosi essenzialmente a protezione del canale di collegamento con il Tevere, ostruito alla foce ma ancora navigabile verso Roma. 
Pur non essendo chiaro quando il processo di insabbiamento bloccò definitivamente gli accessi ai bacini di Claudio e Traiano, questo doveva essere ad uno stadio molto avanzato nel Medioevo; Il periodo di clima più caldo sopravvenuto tra l'800 e il 1200 definito Optimum Climatico medievale avrebbe provocato un parziale scioglimento dei ghiacci polari e continentali col conseguente innalzamento del livello dei mari. È evidente che la presenza del cordone di dune alluvionali litoranee formatesi in età storica per azione del Tevere ha reso difficoltoso il deflusso delle masse d'acqua dei fiumi nel mare. Le dune, oggi scomparse, presentavano dei profili ondulati e dei rilievi alti una ventina di metri, di conseguenza l'impaludamento di tratti di pianura costiera e i processi di interramento della zona con il progressivo allontanamento del Porto di Traiano dal mare.

Sappiamo che nel XV secolo, all'inizio dell'età moderna, l'intero bacino era ormai completamente insabbiato e trasformato in palude.

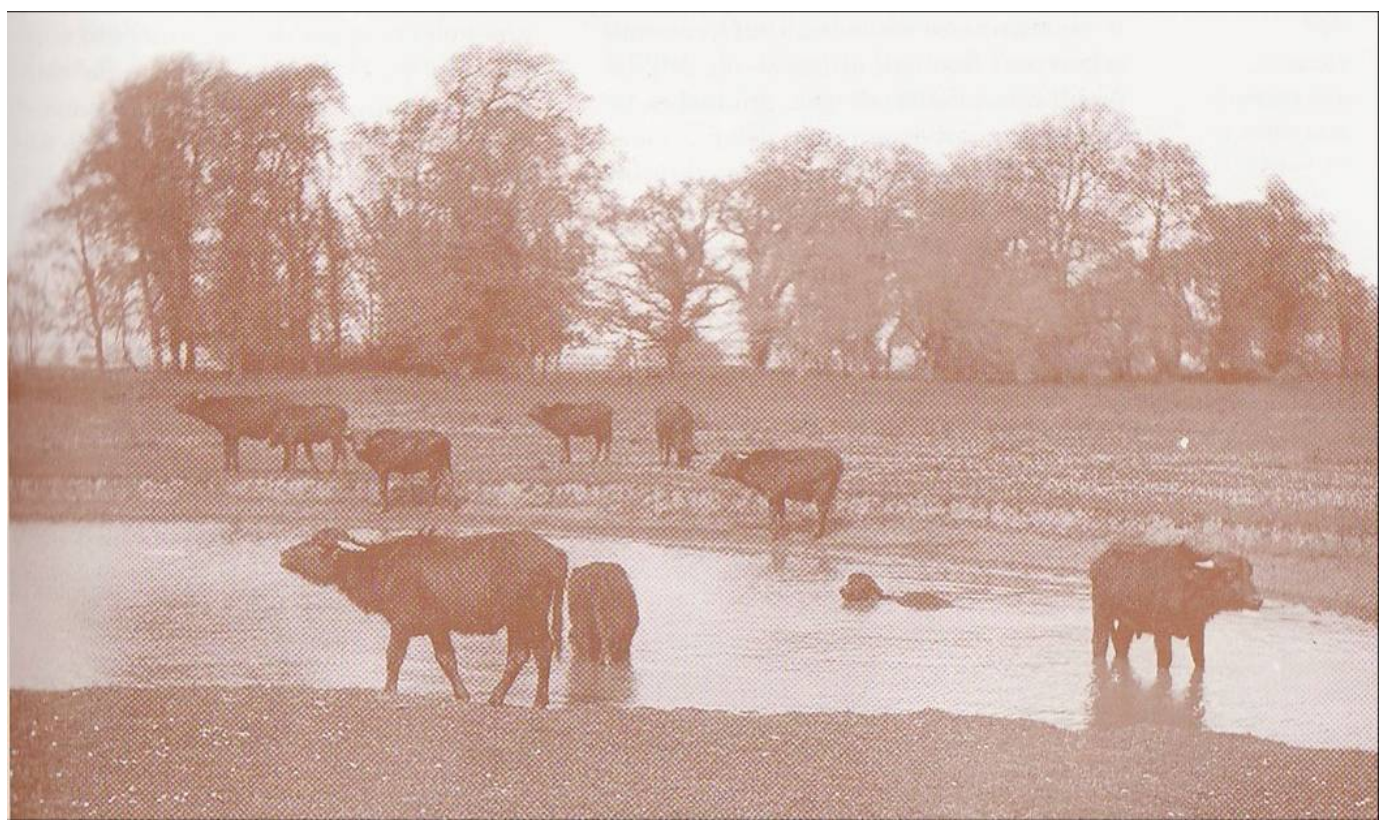

Fig. 3. Foto d'archivio (Comune di Fiumicino).

Nell'800 il paesaggio era formato in prevalenza da aree boschive e terreni paludosi che, vista l'impossibilità dell'acqua stagnante di defluire verso il mare, ricoprivano l'intera zona in modo quasi permanente. Vaste paludi, foreste allagate, foci ampie costituivano l'habitat ideale per una fauna di grande importanza ma anche per la riproduzione delle zanzare anofele responsabili della malaria, malattia che provocava altissima mortalità nella popolazione, soprattutto infantile. L'uso del suolo era per forza di cose prevalentemente destinato a pascolo, alla pesca e solo in minima parte ancora per la raccolta del sale.

In questo desolato e struggente paesaggio, il governo intervenne per facilitare la penetrazione dell'uomo e l'utilizzo delle terre malsane.

I grandi interventi che hanno bonificato tutta la piana tiberina sono stati realizzati a cavallo del XIX e il XX secolo. Hanno sicuramente risolto i problemi di ordine sanitario e agricolo modificando però al contempo la morfologia del suolo, l'ambiente e tutto l'ecosistema che da esso dipendeva. Al prosciugamento delle paludi 
seguì la distruzione della originaria associazione litoranea fatta di lecci e sughere, di farnie, pioppi bianchi, ontani, olmi e frassini. La vegetazione presente lungo il litorale apparteneva soprattutto ai lecceti mesofili: lecci, allori, tini e nelle zone più umide alla serie dei boschi igrofili con salici e pioppi. Oltre alla vegetazione arborea erano presenti gli arbusteti sclerofilli sempreverdi: lentischi, siliquastri, corbezzoli, mirti. $\mathrm{Ne}$ da una descrizione mirabile Plinio il giovane (vissuto proprio negli anni della costruzione dei porti imperiali), nella sua lettera all'amico Gallo descrive la sua villa Laurentina a 17 miglia da Roma e parla dei boschi che vede dalla sua finestra verso il mare.

A questo paesaggio naturale si sostituisce, per ampi tratti di territorio, un paesaggio costruito dall'uomo caratterizzato da una bassa pianura solcata da un ordinato sistema di canali artificiali e da piantumazioni arboree alloctone di grande resistenza e invasività.

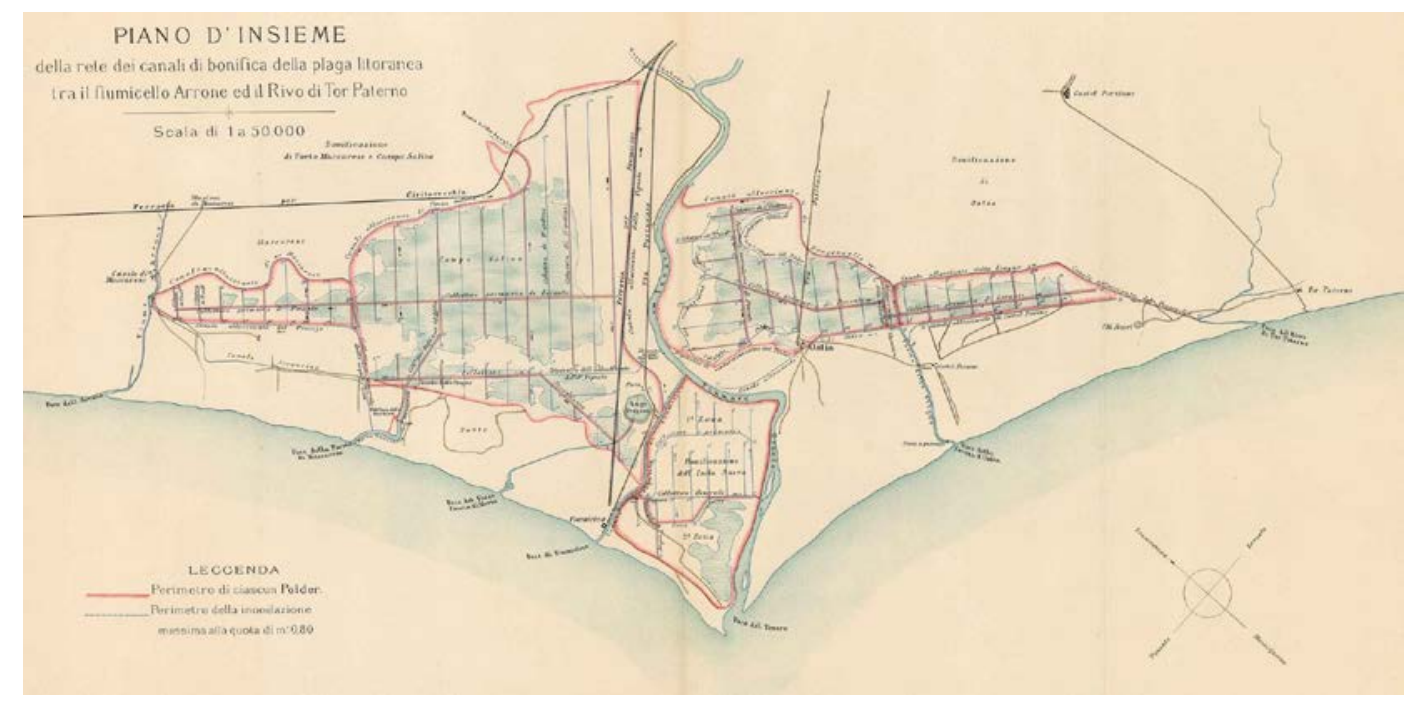

Fig. 4. Rede dei canali di bonifica.

Nel 1924 il principe Giovanni Torlonia diede inizio a quelle opere di bonifica idraulica e di piantumazione che trasformarono radicalmente l'area quale noi possiamo vederla, nelle sue linee essenziali, ancora oggi. L'intento era quello di trasformare dei possedimenti improduttivi in una tenuta agricola modello. L'antico porto esagonale di Traiano fu risanato e utilizzato come bacino per la raccolta delle acque necessarie per l'irrigazione delle nuove piantagioni, distribuite con un sistema di canalizzazioni in cemento ancora visibili nell'area.

Furono utilizzati i lacerti murari degli antichi moli per le fondazioni del grande casale con serbatoio idrico a torretta, e il più piccolo situato poco distante, per assicurare la solidità necessaria ai manufatti, poiché il terreno di sedimentazione poco coeso non dava garanzia di stabilità.

Indagini geognostiche moderne hanno fornito delle indicazioni sulla stratificazione del suolo: carotaggi nell'area del porto di Claudio e in quella di Traiano hanno evidenziato le componenti sabbiose-limose-argillose del terreno ma stratificate in modo diverso a seconda si tratti di riporti marini o fluviali. Tutt'oggi il terreno altamente impermeabile non facilita il deflusso delle acque meteoriche o alluvionali. 
Per ovviare ai ripetuti episodi di allagamento di alcune aree sono state messe in opera recentemente dei drenaggi di superficie per la regimentazione delle acque in esubero sul piano di campagna.

Il patrimonio arboreo, "costruito" in quel periodo, e che può senza dubbio ormai considerarsi storico, ammonta oggi a più di 2000 esemplari.

Le specie presenti sono di tipo comune, specie ben adattabili al clima ed al substrato del luogo, in grado di svolgere compiti ben precisi, ad esempio la tamarix gallica come diaframma di protezione dai venti marini o l'Eucalyptus globulus adatte ad assorbire l'acqua in eccesso nel terreno; il Pinus pinea che, sebbene introdotto sul litorale dall'uomo alla fine del 1600, nelle aree più propriamente con vocazione naturale a macchia mediterranea, era ampiamente diffuso e utilizzato nel territorio nazionale, soprattutto lungo i nuovi assi viari della nuova sistemazione urbanistica di Roma negli anni del governatorato.

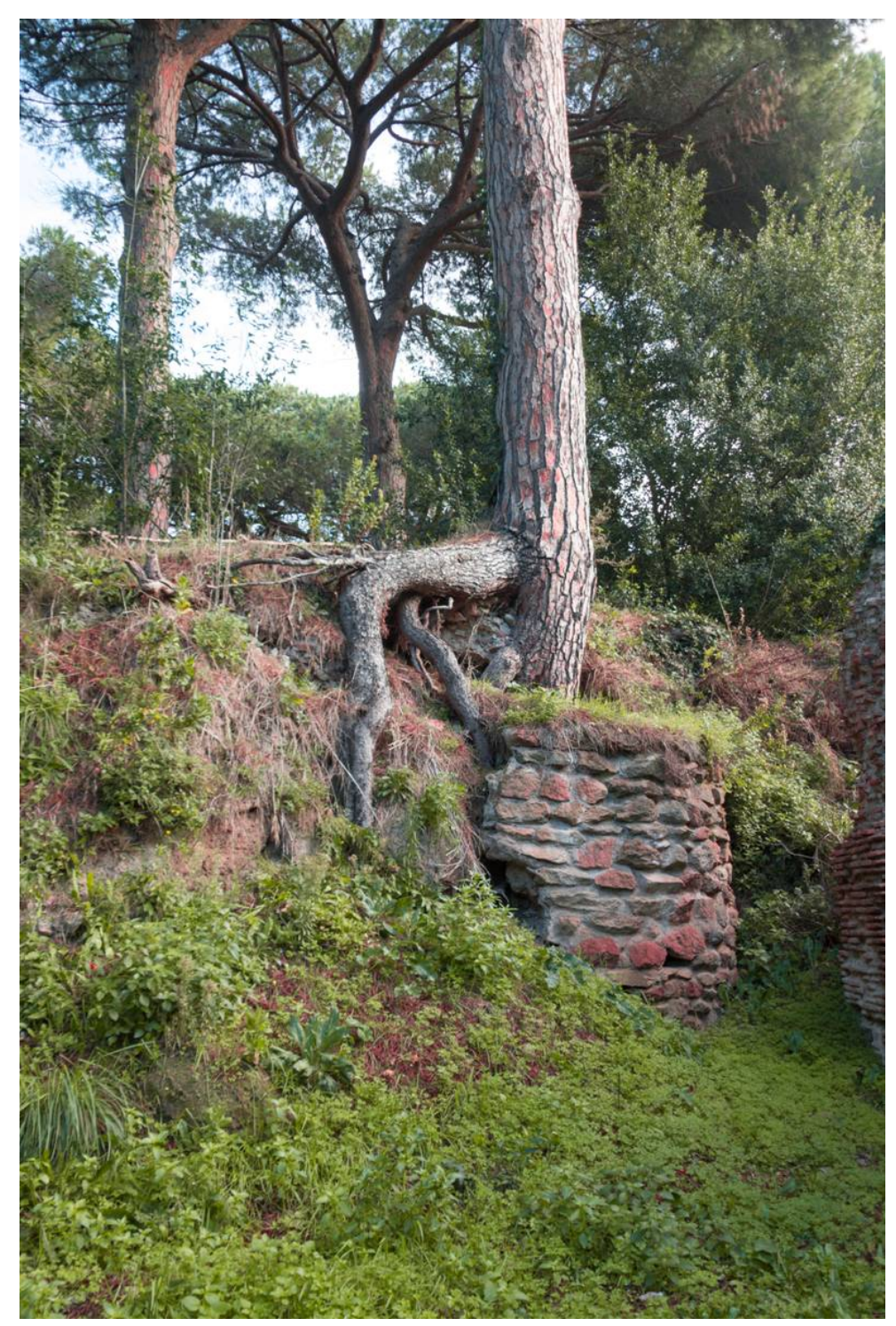

Fig. 5. Pino con tiranti (foto G. STRANO). 
La struttura della tenuta agricola si individua anche nelle linee geometriche ed estetiche dei filari di alberi che costeggiano tutt'oggi i viali: Platanus ibrida, Cupressus sempervirens, Quercus ilex, e i già citati Eucalyptus globulus, Pinus pinea.

Alla tenuta agrícola "modello" è seguito un uso improprio dell'area quale zoo-safari (anni 1970/80) e infine l'abbandono fino al momento dell'acquisizione come terreno Demaniale. (esproprio, prima fase: 12 settembre 1991. seconda fase 1992).

La situazione che si presentava all'inizio dell'intervento di riqualificazione come Parco Archeologico Naturalistico (1997-1998, che comprendono l'area portuale ad esclusione del grande bacino esagonale tutt'oggi ancora di proprietà privata) era quella di un'area pesantemente inselvatichita per la presenza di vegetazione infestante che rendeva pressoché impossibile l'accesso e che crescendo a ridosso o dentro i manufatti antichi ne pregiudicava la stabilità e d'altro lato soffocava altre specie di grande importanza botanica.

Le legnose che rivestono una maggiore pericolosità, qui come in genere nei contesti archeologici per un impatto negativo con i manufatti, sono costituite soprattutto da alcune specie ruderali pioniere, quali Ficus carica, Hedera belix, Robinia pseudo-acacia, Celtis australis ma anche Quercus ilex e Laurus nobilis. (f.to 5)

Parlare di piante infestanti ci inoltra in un percorso particolare dal punto di vista botanico o, in senso più ampio, di conservazione dei beni da tutelare. In accordo con la definizione data dal Terminology Commitee of the Weed Science Society of America: "possono considerarsi infestanti nell'accezione specie vegetali presenti laddove non sono desiderate". Una definizione particolarmente appropriata per le aree archeologiche dove la vegetazione, se non è adeguatamente controllata, può mettere a serio rischio i delicati equilibri. Nel parco rivestono carattere di infestanti piante che in altri contesti, e in alcuni ambiti del parco stesso, sono di grande valenza paesaggistica. Il criterio adottato per la sistemazione è stato quello di consentire la lettura dell'antico impianto portuale nel rispetto e nella valorizzazione dell'importante patrimonio arboreo. Nella prima fase d'intervento sono stati eliminati le superfetazioni e i materiali in abbandono presenti nell'area, a seguire tutti gli alberi morti e quelli con patologie irreversibili.

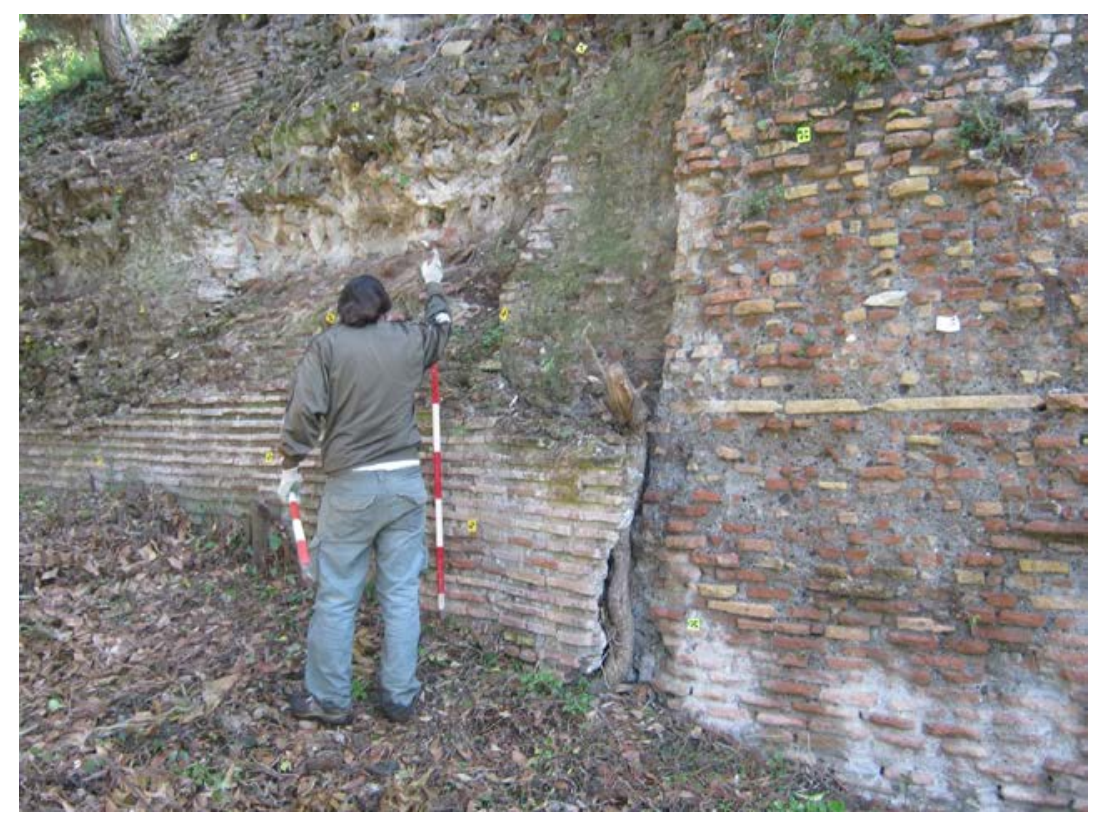

Fig. 6. Problematiche di conservazione (foto G. STRANO). 
Sono state sacrificate piante insistenti sulle murature solo nei casi dove fosse previsto l'intervento di consolidamento e restauro; eliminare le radici infiltrate nei muri o negli intonaci senza intervenire tempestivamente sul recupero del manufatto crea dei percorsi privilegiati per l'acqua piovana e incrementa la disgregazione.

Così anche per la grave lacuna nei magazzini portuali: i piani superiori degli edifici sono andati perduti, si è proceduto al consolidamento delle strutture e al mantenimento delle piante spontanee cresciute sulle creste murarie, frammento prezioso di quella evoluzione biologica naturale potenziale a cui tende un'area non sottoposta ad interventi antropici. Il monitoraggio dello sviluppo radicale e la possibilità di fondi idonei indirizzeranno le scelte future più opportune per la salvaguardia del manufatto archeologico. Inevitabilmente i primi interventi hanno creato una situazione di alterazione degli equilibri ecologici che si erano andati formando soprattutto nell'ultima fase di abbandono. Le alberature avevano sviluppato un forte indirizzo verso l'alto. Le folte chiome fungevano anche da sostegno riducendo così il bisogno di sviluppo dell'apparato radicale. Nel momento dell'alleggerimento delle parti aeree si sono verificati crolli di alberature con difetti nell'impianto radicale. Gli interventi successivi sono stati mirati a garantire la maggior sicurezza possibile in ordine di stabilità della pianta, soprattutto di quelle poste lungo i viali di percorrenza.

La chiave di lettura è stata quella di mantenere una vegetazione bassa a carattere prativo in quegli ambiti dove prima era il mare per consentire, in un'area ormai completamente interrata, la spazialità dello sguardo e l'idea delle acque nel bacino portuale.

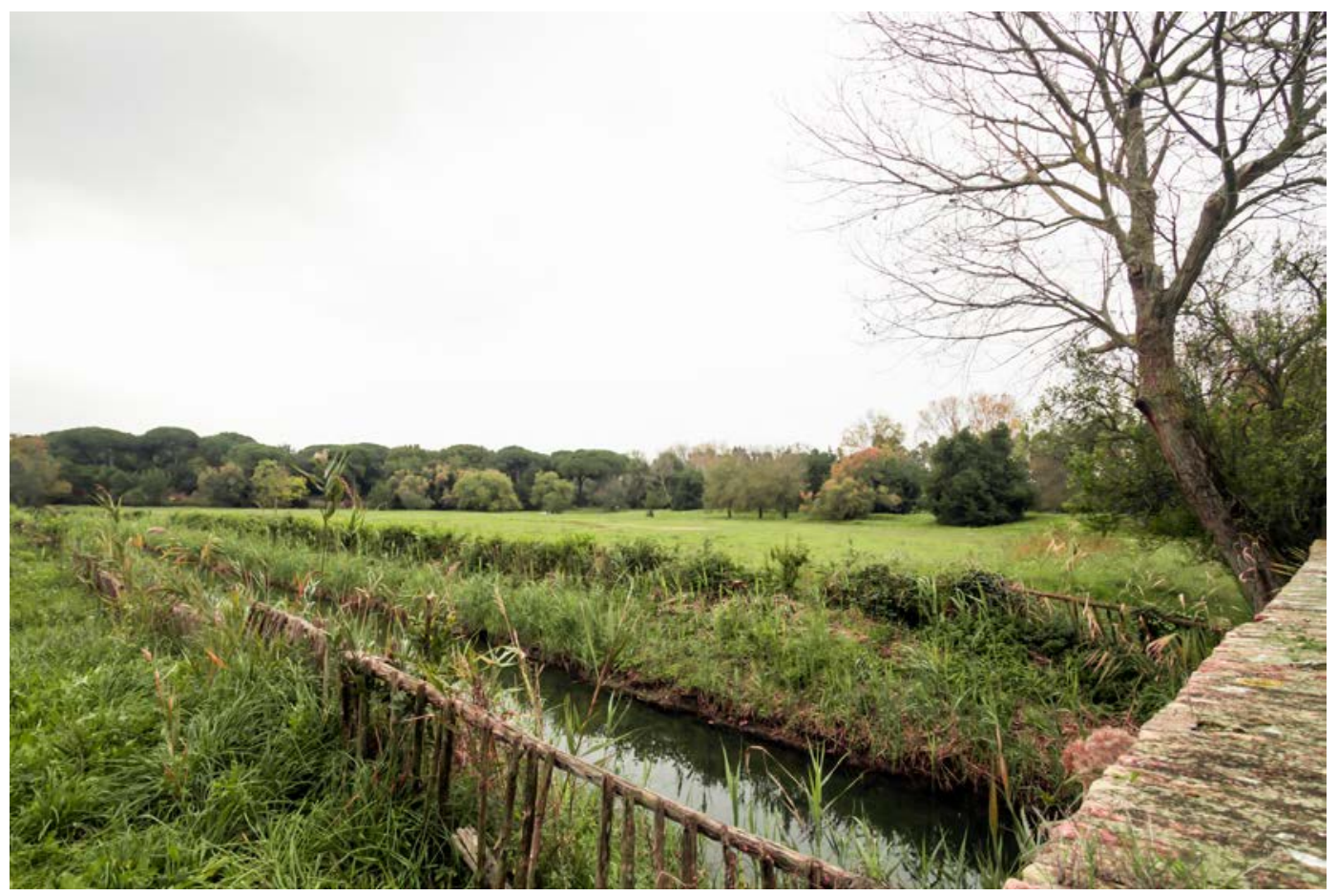

Fig. 7. Panoramica (Foto F. TORTORA). 
Nell'area sono in corso costantemente indagini e interventi archeologici rispettando al massimo le grandi alberature che vengono abbattute solo in caso di grave patologia con rischio di schianti dei rami o dell'intera pianta con pericolo per gli operatori e i fruitori del Parco.

Anche gli interventi di diserbo sono stati e sono molto contenuti, valutati caso per caso tenendo conto di vari fattori quali il grado di degrado del manufatto, il valore estetico ambientale della pianta e mirati all'eliminazione delle piante con alto valore di Indice di Pericolosità della specie.

Si tratta di un indice numerico che esprime sinteticamente, per ciascuna specie vegetale presente nell'area in esame, la pericolosità nei confronti dei manufatti architettonici.

Gli anni di abbandono hanno favorito lo sviluppo di alcune specie non previste nel piano progettuale della tenuta agricola del 1924, ed è uno degli aspetti più interessanti, dal punto di vista botanico, che caratterizzano il sito archeologiconaturalistico. La vegetazione naturale e seminaturale che si trova nel parco, anche se notevolmente depauperata, è strettamente collegata a quella che si trovava nella zona e nelle aree circostanti anche nel passato: farnie, carpini, olmi, tigli, lecci, allori, bagolari ma anche lentischi, corbezzoli e, nelle aree più umide salici e pioppi.

Dal punto di vista strettamente naturalistico gli aspetti più interessanti sono costituiti dai boschi di Quercus ilex, Pistacia lentiscus e Phillyrea, Laurus nobilis e dal biotopo acquatico nella grande darsena, dove è stata mantenuta la vegetazione palustre composta da canne, tife e giunchi, che presenta una biodiversità e un ecosistema di notevole valenza biologica. Dal punto di vista paesaggistico ripropone la visione e la funzione degli antichi stagni che componevano il paesaggio prima della bonifica della fine del XIX secolo; è il punto di sosta degli uccelli migratori e, come già indicato, salvaguardato dalle normative europee.

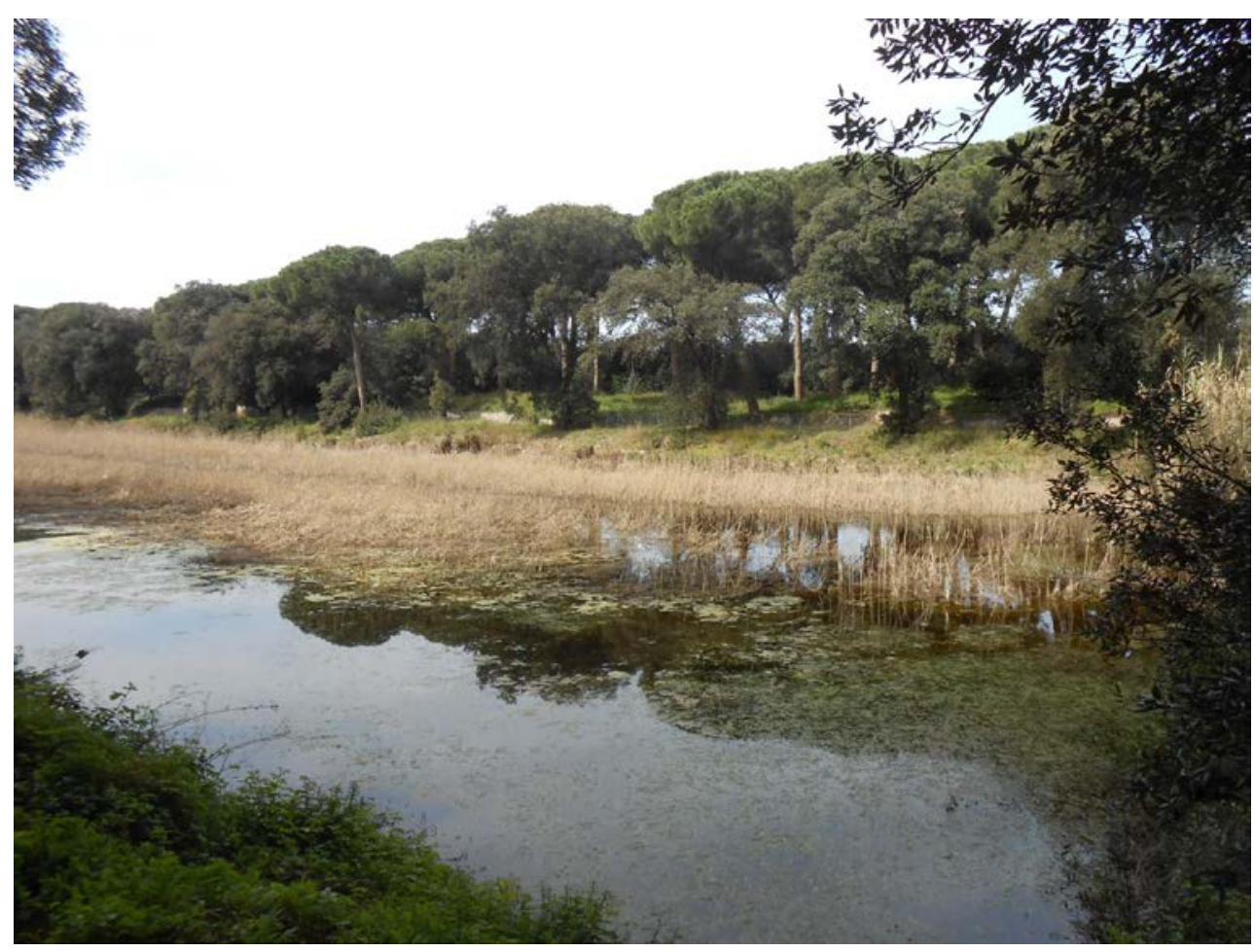

Fig. 8. Darsena (foto G. STRANO). 
Il parco archeologico naturalistico dei Porti di Claudio e Traiano si caratterizza per il forte connubio tra il sistema delle architetture dell'antico impianto portuale allo stato di rudere, il patrimonio arboreo e la presenza degli specchi d'acqua, in una unità armonica resa suggestiva dalle tracce che il tempo ed il degrado hanno impresso sulle antiche vestigia e sul paesaggio. L'esigenza della conservazione e tutela dei monumenti deve legarsi necessariamente ad una corretta ed efficace gestione della vegetazione che analizzi accuratamente modalità di esecuzione, efficacia, costi, ricadute ambientali ma anche l'interesse scientifico-ecologico delle comunità vegetali presenti. Si sta curando altresì un modo e dei tempi di fruizione che non disturbino la fauna presente e al contempo permetta la fruizione e la lettura dell'intero bacino portuale: il parco di Portus, il molo settentrionale e l'area archeologica c.d. di Monte Giulio

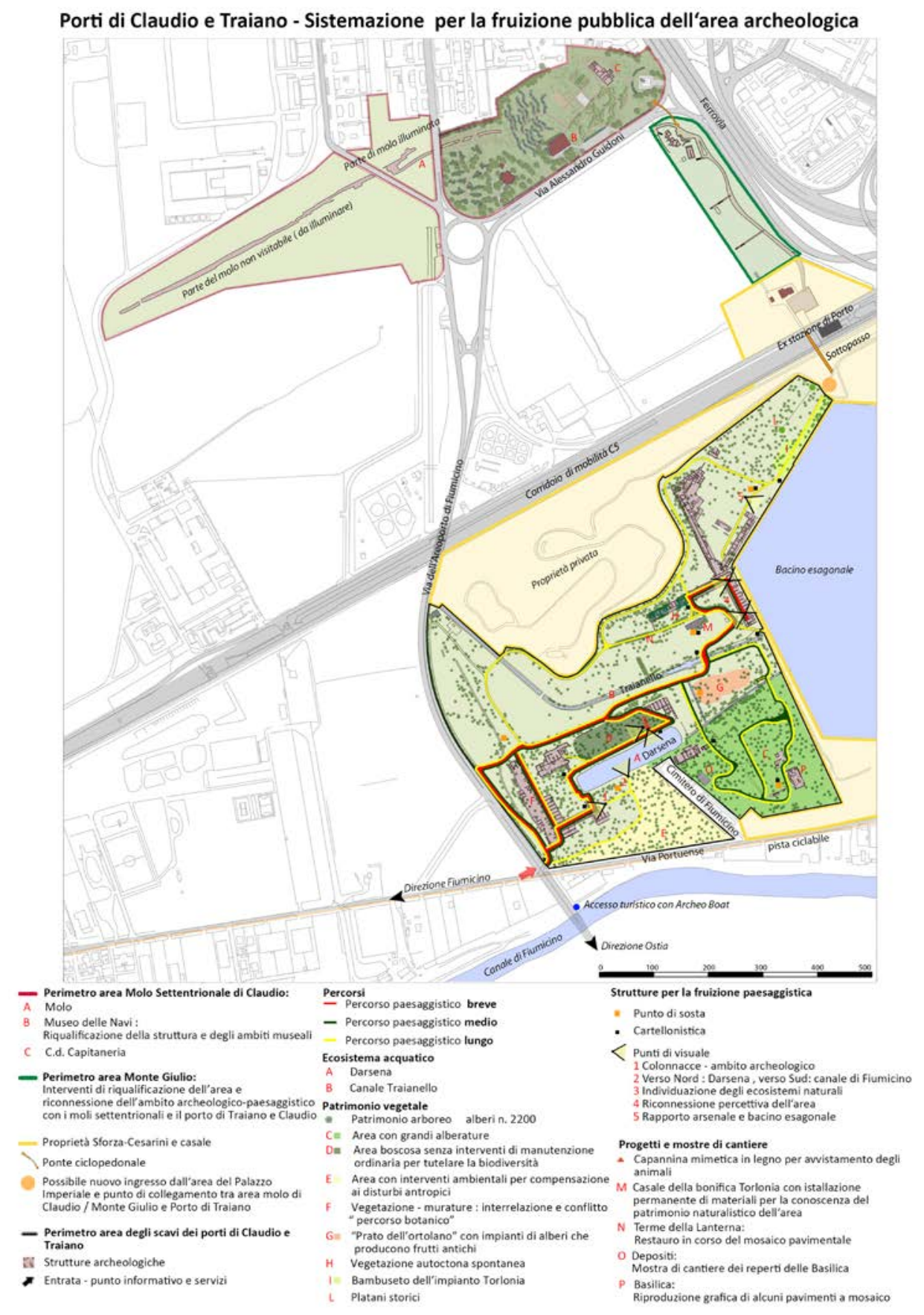

Fig. 9. Plan generale (G. Strano, I. Patriarca, J. PetTinari) 